\title{
In Vitro Antioxidant Capacity of Opuntia spp. Fruits Measured by the LOX-FL Method and its High Sensitivity Towards Betalains
}

\author{
Andrea Gómez-Maqueo ${ }^{1,2,3} \cdot$ Mario Soccio $^{4} \cdot$ M. Pilar Cano ${ }^{1}$ (i)
}

Accepted: 19 July 2021 / Published online: 7 August 2021

(c) The Author(s) 2021

\begin{abstract}
Current in vitro methodologies neglect or subestimate the contribution of betalains to antioxidant capacity in foods because they do not reflect their in vivo biological mechanisms. In this study, we assessed the sensibility of the lipoxygenasefluorescein (LOX-FL) method towards betalains, phenolic compounds and ascorbic acid from Opuntia spp. fruits; and (ii) the antioxidant capacity of peel and pulp extracts from Opuntia ficus-indica L. Mill (var. Fresa, Colorada and Blanco) and Opuntia stricta var. Dillenii; by comparing the LOX-FL method to traditional antioxidant methods (ORAC and TEAC). The spectrophotometric monitoring of the LOX-FL reaction avoided interference caused by betalain pigments. Indicaxanthin and betanin showed high antiperoxidative and radical scavenging mechanisms in the LOX-FL assay. O. stricta var. Dillenii tissues the highest antioxidant capacity which correlated with betanin content. ORAC and TEAC antioxidant methods were less sensible towards betalain antioxidant activity. To our knowledge, this is the first time the LOX-FL antioxidant method has been used on betalains and betalain-rich foods.
\end{abstract}

Keywords Antioxidant capacity $\cdot$ LOX-FL assay $\cdot$ Opuntia ficus-indica $\cdot$ Opuntia stricta var. Dillenii $\cdot$ Betalains $\cdot$ Phenolic compounds

\section{Abbreviations \\ LOX-FL Lipoxygenase-fluorescence \\ ORAC Oxygen radical absorbance capacity \\ TEAC Trolox equivalent antioxidant capacity}

\section{Introduction}

Dietary antioxidants are of interest from biological, medical, and nutritional perspectives because they contribute to the reduction of risk of diseases derived from metabolic syndrome. Betalains are water soluble natural colorants composed of a nitrogenous core structure called betalamic acid. They are classified in two groups, betacyanins (red-violet colors) and betaxanthins (yellow-orange colors) depending on the nature of the added residue. The few edible known sources of betalains are red and yellow beetroot (Beta vulgaris L. ssp. vulgaris), coloured Swiss chard (Beta vulgaris L. ssp. cicla), grain or leafy amaranth (Amaranthus sp.) and cactus fruits, such as those of Opuntia and Hylocereus genera [1].

Edible cactus from the Opuntia genus are nutritious sources of healthy foods adaptable to expanding regions of hot climate [2]. The most widely consumed species from the Opuntia genus is prickly pear (Opuntia ficus-indica L. Mill.), which is widely cultivated in Latin America, Africa, and in the Mediterranean region (Spain, Italy, Morocco and Algeria). Prickly pear fruits are juicy, colored, fruits rich in betalains (betanin, indicaxanthin, portulacaxanthin, vulgaxanthin, betanidin) and phenolic compounds (piscidic acid, 
isorhamnetin glycosides, quercetin glycosides and kaempferol glycosides) [3, 4]. The lesser studied variety, Opuntia stricta var. Dillenii, is native to Central America and may also be found in the Mediterranean region, China, and North Africa. They are referred to as sweet- or wild prickly pear fruits and have recently been characterized in terms of betalains (betanin, phyllocactin, betanidin, and neobetanin) and phenolic compounds (piscidic acid, eucomic acid, and isorhamnetin glycosides) [5, 6].

Betalains have efficient antioxidant action against LOO', $\mathrm{LO},{ }^{1} \mathrm{O} 2,{ }^{\circ} \mathrm{OH}$ and can prevent lipid peroxidation when incorporated to liposomal bilayers submitted to AAPH [7-10]. They are also good peroxidase substrates for myeloperoxidase and work as one-electron reductants of its redox intermediates [11]. Furthermore, the antioxidant effects of these pigments can prevent or delay the progress of neural death in Parkinson's disease [12] in addition to possessing analgesic activity [13].

However, betalains have low sensitivity to widely used antioxidant assays (e.g., ORAC and TEAC) since these in vitro assays do not reflect their antioxidant effects in vivo. Limitations related to the chemistry of these assays are that they are only able to evaluate scavenging capacity against specific types of radical species (some which are not physiological and biologically relevant) and fail to evaluate other important antioxidant effects. Soybean lipoxygenase-based methods could be suitable alternatives for assessing the antioxidant capacity of betalains because they simultaneously detect the scavenging of physiological radical species, iron ion reducing and chelating activities, and inhibition of the pro-oxidant apoenzyme [14].

The objective of this study was to assess (i) the sensibility of the lipoxygenase-fluorescein (LOX-FL) method towards betalains, phenolic compounds and ascorbic acid from Opuntia spp. fruits; and (ii) the antioxidant capacity of peel and pulp extracts from Opuntia ficus-indica L. Mill (var. Fresa, Colorada and Blanco) and Opuntia stricta var. Dillenii; by comparing the LOX-FL method to traditional antioxidant methods (ORAC and TEAC). We expect to shed new light on the antioxidant mechanisms of betanin and indicaxanthin and provide an in vitro methodology for assessing the antioxidant capacity of betalain-rich foods.

\section{Materials and Methods}

\section{Isolated Standards}

Betanin-rich extract was obtained from commercial beetroot and purified in a Sephadex L20 resin to obtain the betanin standard [15]. Indicaxanthin was semi-synthesized from purified betalain by raising the $\mathrm{pH}$ with ammonia to obtain betalamic acid and by reacting with proline [15]. Piscidic acid was purified by semi-preparative high-performance liquid chromatography (HPLC) from extracts of Opuntia ficus-indica peels [16]. Standards for isorhamnetin glucosylrhamnosyl-rhamnoside (IG1), isorhamnetin glucosyl-rhamnosyl-pentoside (IG2), isorhamnetin glucosyl-pentoside (IG4) and isorhamnetin glucosyl-rhamnoside (IG5) were isolated from Opuntia cladodes [17]. All isolated standards were analyzed by HPLC to determine their purity: betalains (95-100\%), piscidic acid (97\%) and isorhamnetin glycosides (84-87\%). Ascorbic acid was purchased from Sigma-Aldrich (99\%).

\section{Plant Material}

Colorada and Fresa prickly pear (Opuntia ficus-indica L. Mill.) fruits were obtained from Fasnia (Tenerife, Canary Islands, Spain; 28 $2^{\circ} \mathrm{N}, 16^{\circ} 4^{\prime} \mathrm{W} ; 446$ masl). Blanco prickly pear (Opuntia ficus-indica L. Mill.) fruits were obtained from Buenavista del Norte (Tenerife, Canary Islands, Spain; $28^{\circ} 2^{\prime} \mathrm{N}, 16^{\circ} 5^{\prime} \mathrm{W} ; 127$ masl). Wild prickly pears (Opuntia stricta var. Dillenii) were obtained from Tinajo (Lanzarote, Canary Islands, Spain; 29 $3^{\circ} \mathrm{N}, 1^{\circ}{ }^{\prime}$ 'W; 209 masl). Thornless fruits were washed and selected according to uniform maturity, size and no defects. Their physicochemical characteristics were determined in ten fruits of each variety (Supplementary Table S1). Prickly pears were separated into peels and pulps, cut into small pieces $(20 \times 20 \mathrm{~mm})$, vacuumsealed in polyethylene bags, frozen with liquid nitrogen and freeze-dried. They were pulverized (Grindomix GM200, Retsch, Germany) to a fine particle size $(<2 \mathrm{~mm})$ and seeds were removed. Pulverized samples were vacuum-sealed and stored at $-20^{\circ} \mathrm{C}$ until analysis.

\section{Extracts}

Prickly pear extracts were obtained from freeze-dried and pulverized tissues by extracting three times with methanol:water $(1: 1, \mathrm{v}: \mathrm{v})$, one more time with pure methanol, and by evaporating the methanol from the obtained supernatants to obtain the aqueous extract [16]. The aqueous extracts were analyzed to quantify betalains, phenolic compounds and ascorbic acid and they were used to assess in vitro antioxidant capacity by different methods.

\section{Quantification of Total Betalains by Spectrophotometry}

Extracts were diluted and betalains were quantified spectrophotometrically [16] using a SmartSpec Plus BIO-RAD ${ }^{\circledR}$ spectrophotometer and expressed in terms of betaxanthin and betacyanin equivalents by measuring the absorbance at 483 and $535 \mathrm{~nm}$, respectively. 


\section{Quantification of Betalains and Phenolic Compounds by HPLC}

Betalains and phenolic compounds were determined simultaneously by high performance liquid chromatography according to reported methodology $[3,16]$. The main bioactive compounds were identified by their retention time, UV-visible and mass spectral data compared to those of purified, semi-synthesized and commercial standards (Supplementary Table S3) and quantified using their respective calibration curves. Ascorbic acid was determined by the microplate-adapted colorimetric ascorbate assay [16].

\section{Lipoxygenase-Fluorescein (LOX-FL) Antioxidant Capacity}

Experiments were performed using the LOX/RNO [14] and LOX-FL [18] methods. The LOX/RNO reaction was spectrophotometrically monitored by measuring the absorbance decrease of 4-nitroso- $N, N$-dimethylaniline (RNO) at $440 \mathrm{~nm}$ in the course of linoleate hydroperoxidation by soybean LOX-1 isoform. The LOX-FL method was performed by fluorometrically monitoring the fluorescein quenching $\left(\lambda_{\mathrm{ex}}=485 \mathrm{~nm} ; \lambda_{\mathrm{em}}=515 \mathrm{~nm}\right)$ associated to LOX-1-catalysed linoleate peroxidation.

Based on these results, a protocol for studying the antioxidant capacity of Opuntia extracts by the LOX-FL assay was defined. This method involved the spectrophotometric monitoring of the LOX-FL reaction at $485 \mathrm{~nm}$ [18] and slight modifications. Fluorescein bleaching was monitored using a spectrophotometer (Specord 210 plus, AnalytikJena, Germany) in a reaction mixture (1 mL) containing $100 \mathrm{mM}$ Na-borate buffer $\mathrm{pH}$ 9.0, $400 \mu \mathrm{M}$ Na-linoleate, $1 \mu \mathrm{L}$ Tween 20 per $\mu \mathrm{mol}$ linoleate and $4.5 \mu \mathrm{M}$ FL. The linoleate solution was prepared as reported previously. [14] The reaction was started by adding $0.5 \mathrm{EU}$ of soybean lipoxygenase. Measurements were carried out in both the absence (control) and presence of sample (extract or standard). The rate of the reaction expressed as $\Delta \mathrm{A}_{485} \bullet \mathrm{min}^{-1}$, was calculated as the highest slope to the experimental curve. The lag phase was calculated as the time occurring between enzyme addition to the test sample and the start of the reaction.

The inhibition of the LOX-FL reaction was determined by calculating the decrease of the rate of the fluorescein bleaching in the presence of sample (extract or standard) $\left(\mathrm{v}_{\mathrm{a}}\right)$ with respect to the control $\left(\mathrm{v}_{\mathrm{c}}\right)$, according to the equation: Inhibition $(\%)=\left[1-\left(\mathrm{v}_{\mathrm{a}} / \mathrm{v}_{\mathrm{c}}\right)\right] \bullet 100$. Antioxidant capacity was calculated by means of a dose-response curve obtained with Trolox by plotting the decrease of the rate of fluorescein bleaching as a function of the Trolox concentration according to the equation: Inhibition $(\%)=4.8594$ [Trolox] +11.6 $(\mathrm{r}=0.9942$, $p<0.001$ ), where the concentration of Trolox was $2-10 \mu \mathrm{M}$. Since both the rate and the lag phase of the LOX-FL reaction were affected by methanol, when isolated isorhamnetin glycosides and piscidic acid (reconstituted in methanol) were evaluated, a constant volume of $50 \mu \mathrm{L}$ of methanol was also maintained in the control.

\section{TEAC Antioxidant Capacity}

The TEAC assay was analyzed spectrophotometrically at $734 \mathrm{~nm}$ [19]. The radical cation ABTS + (2,2'-azinobis-3-ethylbenzothiazoline-6-sulfonic acid) was generated by ABTS oxidation with potassium persulfate. The ABTS $\bullet+$ solution was diluted with $5 \mathrm{mM}$ Na-phosphate buffer at $\mathrm{pH} 7.4$ to obtain an initial absorbance value at $734 \mathrm{~nm}\left(\mathrm{~A}_{734}\right)$ of $0.70 \pm 0.20$. The assay contained $1.0 \mathrm{~mL}$ of the ABTS + diluted solution, the sample (extract or standard), and Na-phosphate buffer ( $\mathrm{pH}$ 7.4) to obtain a final volume of $1.1 \mathrm{~mL}$ assay. Absorbance at $734 \mathrm{~nm}$ was read 5 min after. The decrease of absorbance (\%) with respect to the blank was used to quantify antioxidant capacity using a concentration-response Trolox curve 10-150 $\mu \mathrm{M}$.

\section{ORAC Antioxidant Capacity}

The ORAC protocol [20] was performed by measuring fluorescence degradation in a 96-well microplate. Every working well of a 96-well plate containing the assay mixture (final volume $0.2 \mathrm{~mL}$ ) consisting of $75 \mathrm{mM}$ Na-phosphate buffer ( $\mathrm{pH}$ 7.4), $7 \mu \mathrm{M}$ fluorescein and an appropriate volume of sample (extract or standard). The reaction was started by adding $46 \mathrm{mM}$ AAPH (2,2'-azobis (2-amidinopropane) dihydrochloride) in the well. Fluorescence intensity decay was monitored once every minute during $60 \mathrm{~min}$ at $37^{\circ} \mathrm{C}$. Monitoring was done at $485 \mathrm{~nm}$ excitation and $530 \mathrm{~nm}$ emission wavelengths. To quantify antioxidant capacity, the difference between the area under the fluorescence decay kinetic curve (area under curve, AUC) of sample and the AUC of the blank was calculated. Antioxidant capacity was determined using a Trolox dose-response curve $(10-80 \mu \mathrm{M})$ repeated each time the assay was performed.

\section{Statistical Analysis}

Results were expressed as mean \pm standard deviation $(n=3)$. Significant differences were calculated by one-way analysis of variance (ANOVA), followed by post hoc Duncan's test $(p<0.05)$. Pearson's correlation coefficients $(r)$ were determined $(* p \leq 0.05, * * p \leq 0.001$, bilateral, $n=24)$. Statistical analysis was determined with SPSS Statistics 23.0 (IBM Corp, Armonk, USA). 


\section{Results and Discussion}

\section{Characterization of Opuntia spp. Fruit Extracts by HPLC}

Extracts from Opuntia ficus-indica (var. Colorada, Fresa and Blanco) and Opuntia stricta var. Dillenii peels and pulps were characterized in terms of betalains, phenolic compounds and ascorbic acid (Supplementary Table S2). The extensive betalain and phenolic profile in $O$. ficus-indica fruits (var. Fresa, Colorada and Blanco) from the Canary Islands has been previously determined [3] and the $O$. stricta var. Dillenii betalain and phenolic profile was tentatively evaluated in our laboratory in this work.

\section{Suitability of Soybean Lipoxygenase-Based Antioxidant Methods}

The suitability of the LOX-FL reaction was assessed by evaluating its sensitivity to Opuntia spp. fruit extracts and isolated betalains. We studied the interference of these pigments (due to their spectral properties) by LOX-FL and LOX/RNO methodologies (Fig. 1). Following the traditional LOX-FL method (fluorometric monitoring), a high concentration of betalains ( $\lambda_{\text {abs }} 480$ and $530 \mathrm{~nm}$ ) may quench fluorometric values $\left(\lambda_{\text {exc }} 485 \mathrm{~nm}\right.$ and $\lambda_{\text {emi }} 515 \mathrm{~nm}$ ) during the assay (Fig. 1A). Meanwhile, using the LOX/RNO assay (Fig. 1B) betalains caused false positive values by increased the absorbance of the reaction.

The LOX-FL reaction monitored spectrophotometrically at $485 \mathrm{~nm}$ was the best method to assess the antioxidant capacity of betalains and Opuntia spp. extracts (Fig. 1C). This method has been previously used to evaluate the kinetic properties of the soybean-lipoxygenase reaction [18], being able to replace the detection by fluorimetry. Interestingly, we found that this approach required lower amounts of betalains compared to the LOX/RNO method, by reducing the interference on the assay which was negligible.

\section{Antioxidant Capacity by the LOX-FL Assay}

Isolated betalain, phenolic compound and ascorbic acid standards were individually assessed (Fig. 2) by the LOXFL antioxidant method. The LOX-FL reaction consists of two stages [14]: i) a lag phase, representing the time necessary to consume the oxygen in the reaction mixture due to the primary LOX-1 reaction of linoleate hydroperoxidation (aerobic cycle), and ii) a bleaching phase of fluorescein, due to the LOX-1 mediated generation of physiologically relevant radical species (mainly alkoxyl, peroxyl and hydroxyl radicals), occurring when anaerobiosis is
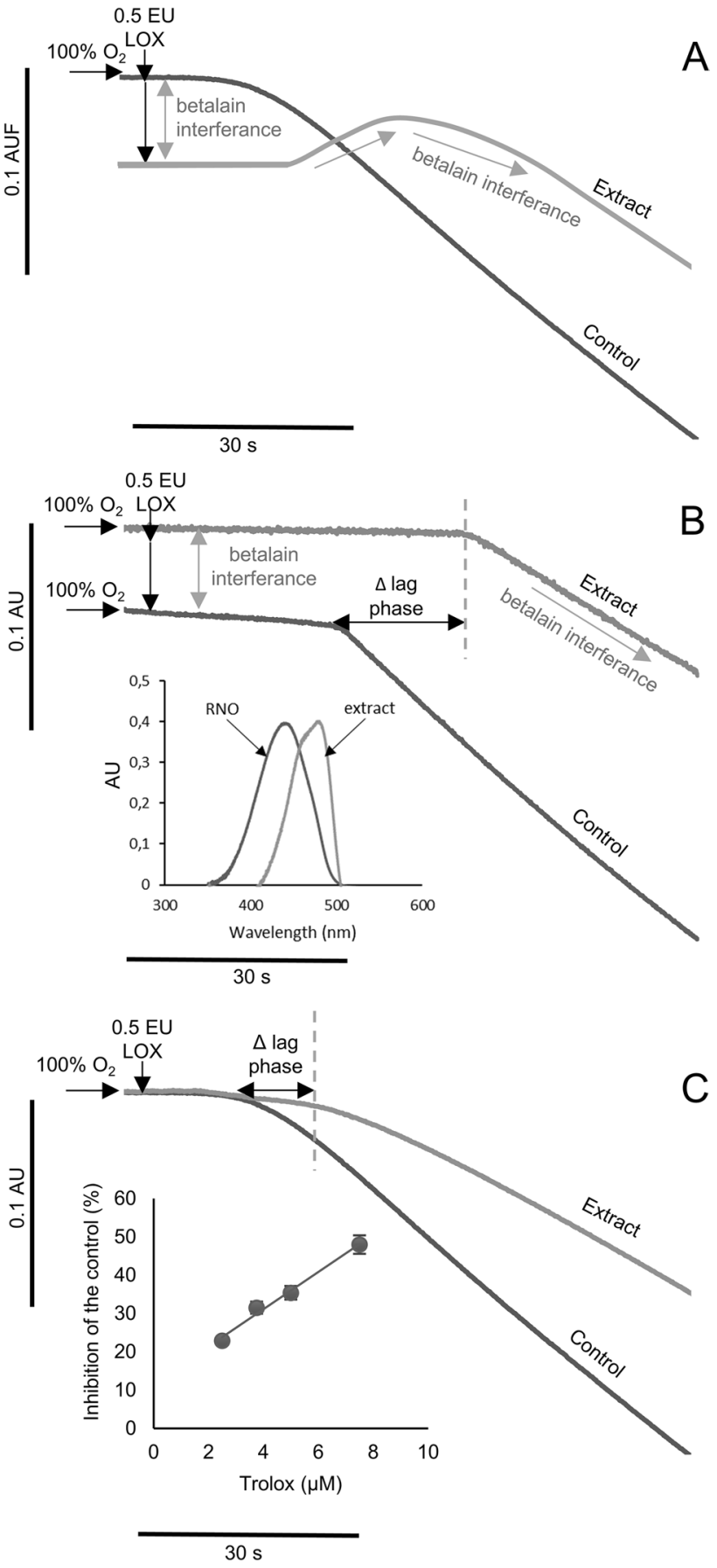

Fig. 1 Comparison of soybean-lipoxygenase methods in betalain-rich extracts and standards. (A) LOX-FL method (fluorometric monitoring), (B) LOX/RNO method (spectrophotometric monitoring) and RNO and extract UV-Vis spectra, (C) LOX-FL method (spectrophotometric monitoring) and Trolox calibration curve. $\Delta$ lag phase: lag phase change in the presence of extract

reached in the assay mixture (anaerobic cycle). All standards showed a positive linear dependence between reaction inhibition and compound concentration. However, the different behavior with respect to the lag phase and bleaching 
Fig. 2 Inhibition of the LOX1-dependent fluorescein bleaching by (A) betanin, (B) indicaxanthin, (C) ascorbic acid, (D) piscidic acid, (E) isorhamnetin glucosyl-rhamnosyl-rhamnoside (IG1), (F) isorhamnetin glucosyl-rhamnosyl-pentoside (IG2), (G) isorhamnetin glucosyl-pentoside (IG4) and (H) isorhamnetin glucosyl-rhamnoside (IG5)
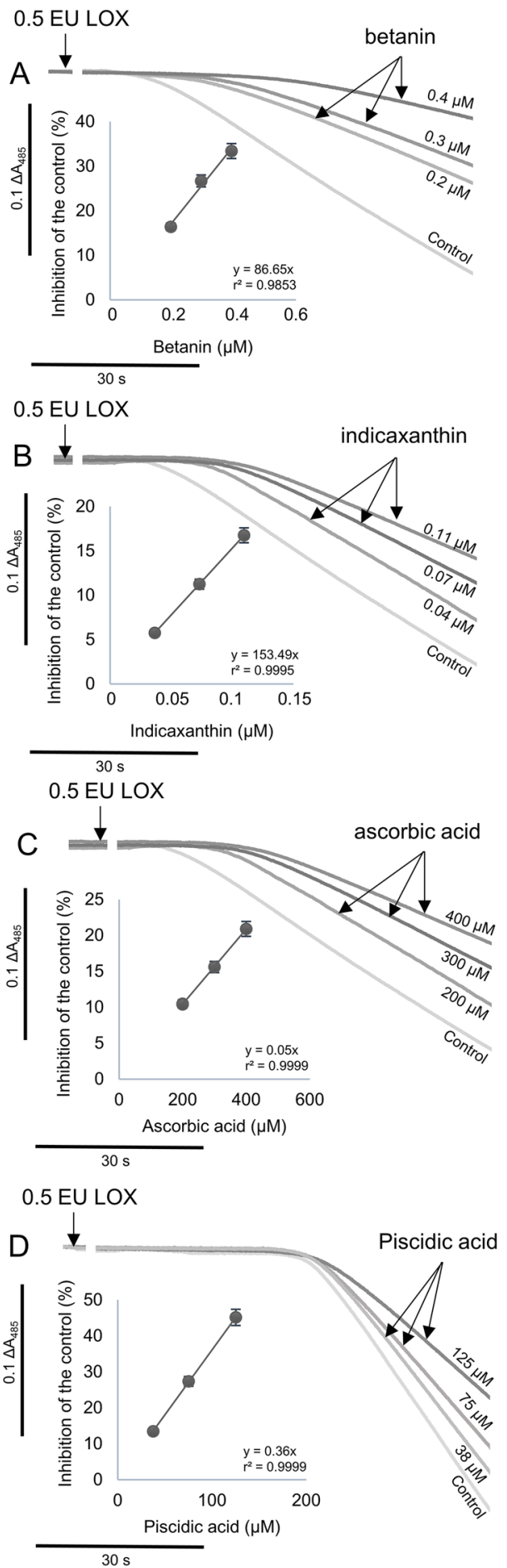
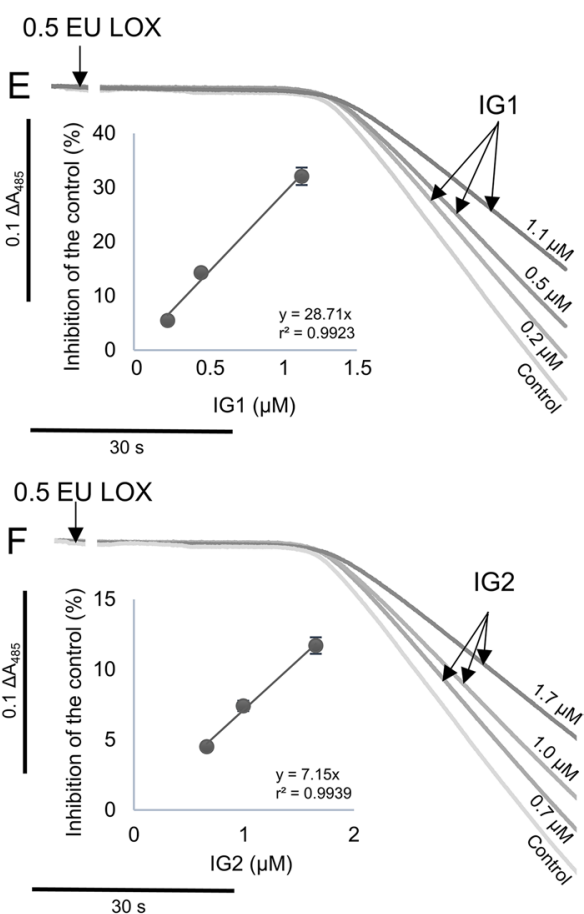

0.5 EU LOX
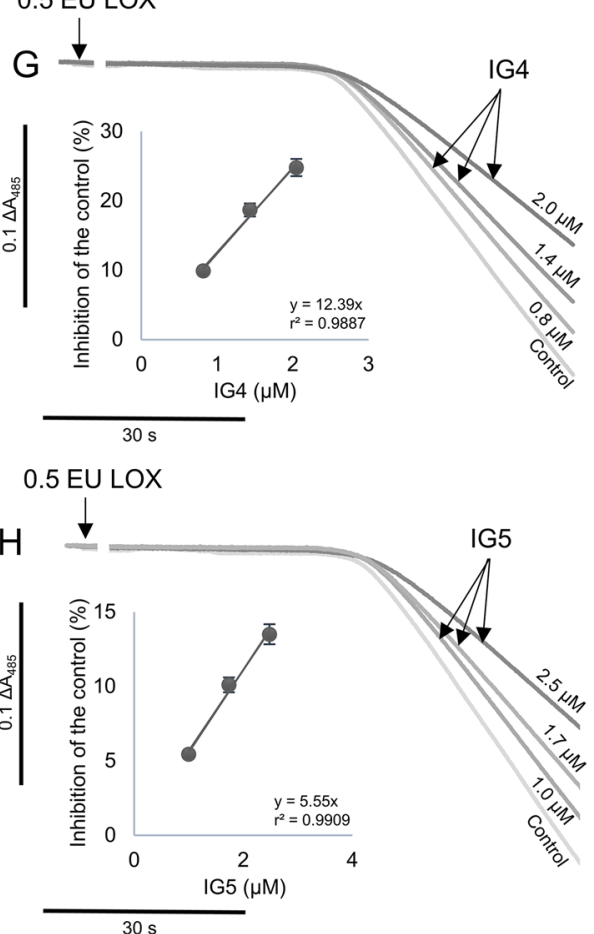

rate of fluorescein strongly suggested different antioxidant actions.

On one hand, betalains (betanin, indicaxanthin) and ascorbic acid inhibited the rate of the fluorescein bleaching (anaerobic reaction), showing radical scavenging activity. Additionally, they increased the lag phase of the reaction (aerobic reaction) evidencing their antiperoxidative action (Fig. 2A-C; Table 1). On the other hand, phenolic compounds (piscidic acid and isorhamnetin glycosides) inhibited the reaction rate by showing high radical scavenging activity but did not affect the lag phase of the reaction (Fig. 2D-H).

In Table 1, the antioxidant capacity of pure indicaxanthin, betanin, isorhamnetin glycosides (IG1, IG2, IG4 and IG5), piscidic and ascorbic acids determined by the 
Table 1 Antioxidant capacity of individual standards (betalains, phenolic compounds and ascorbic acid) by means of the LOX-FL method

\begin{tabular}{|c|c|c|c|c|}
\hline \multirow[b]{2}{*}{ Pure compound } & \multicolumn{2}{|l|}{ LOX-FL } & \multirow{2}{*}{$\begin{array}{l}\text { ORAC }^{2} \\
\mu \mathrm{mol} \text { Trolox eq/mg }\end{array}$} & \multirow{2}{*}{$\begin{array}{l}\text { TEAC } \\
\mu \mathrm{mol} \text { Trolox eq/mg }\end{array}$} \\
\hline & $\mu \mathrm{mol}$ Trolox eq/mg & $\Delta$ lag phase ${ }^{1}$ & & \\
\hline \multicolumn{5}{|l|}{ Betalains } \\
\hline Indicaxanthin & $104.00 \pm 1.98^{\mathrm{a}}$ & +++ & $17.32 \pm 0.68^{\mathrm{c}}$ & $9.04 \pm 2.10^{\mathrm{a}}$ \\
\hline Betanin & $32.27 \pm 1.40^{\mathrm{b}}$ & ++ & $10.92 \pm 0.44^{\mathrm{b}}$ & $5.17 \pm 0.52^{\mathrm{a}}$ \\
\hline \multicolumn{5}{|l|}{ Phenolic acid } \\
\hline Piscidic acid & $0.290 \pm 0.002^{\mathrm{ef}}$ & - & $3.56 \pm 0.16^{\mathrm{a}}$ & $6.43 \pm 0.74^{\mathrm{a}}$ \\
\hline \multicolumn{5}{|l|}{ Flavonoid glycosides } \\
\hline IG1 (Isorhamnetin glucosyl-rhamnosyl-rhamnoside) & $7.64 \pm 0.99^{c}$ & - & $34.96 \pm 1.4^{\mathrm{e}}$ & $122.7 \pm 8.59^{\mathrm{c}}$ \\
\hline IG2 (Isorhamnetin glucosyl-rhamnosyl-pentoside) & $1.90 \pm 0.09^{\mathrm{e}}$ & - & $30.24 \pm 1.2^{\mathrm{d}}$ & $139.56 \pm 6.98^{c}$ \\
\hline IG4 (Isorhamnetin glucosyl-pentoside) & $3.94 \pm 0.23^{\mathrm{d}}$ & - & $27.76 \pm 1.12^{\mathrm{d}}$ & $124.78 \pm 13.20^{\mathrm{c}}$ \\
\hline IG5 (Isorhamnetin glucosyl-rhamnoside) & $1.79 \pm 0.12^{\mathrm{e}}$ & - & $18.00 \pm 0.72^{\mathrm{c}}$ & $72.95 \pm 3.54^{\mathrm{b}}$ \\
\hline \multicolumn{5}{|l|}{ Organic acid } \\
\hline Ascorbic acid & $0.0610 \pm 0.0003^{f}$ & + & $3.04 \pm 0.12^{\mathrm{a}}$ & $8.95 \pm 1.00^{\mathrm{a}}$ \\
\hline
\end{tabular}

Means \pm standard deviation $(n=3)$. Different letters indicate statistically significant differences $(p \leq 0.05)$ according to Duncan's test. ${ }^{1}$ Relative lag phase change (s/mg standard). ${ }^{2}$ Reported in Gómez-Maqueo et al. (2019). LOX-FL values correspond to the experimental data shown in Fig. 2

LOX-FL antioxidant assay (spectrophotometric monitoring) and by ORAC and TEAC antioxidant methods are shown.

In the LOX-FL method, indicaxanthin had the highest antioxidant capacity (104 $\mu \mathrm{mol}$ Trolox eq./mg) followed by betanin ( $32 \mu \mathrm{mol}$ Trolox eq./mg). Though considerably lower than betalains, isorhamnetin glycosides also showed high LOX-FL antioxidant capacity. Piscidic acid was the less active phenolic compound. A low antioxidant capacity value was observed for ascorbic acid. Furthermore, the lag phase of the reaction was affected by betanin, indicaxanthin and ascorbic acid. These results agree with previous findings that show the antioxidant performance of betalains can be higher than that of several flavonoids, ascorbic acid, and tocopherols [21].

Regarding the ORAC antioxidant capacity assay, isorhamnetin glycoside IG1 showed the highest antioxidant capacity (35 $\mu \mathrm{mol}$ Trolox eq./mg) followed by IG2 and IG4 $(28-30 \mu \mathrm{mol}$ Trolox eq./mg) and IG5 (18 $\mu \mathrm{mol}$ Trolox eq./mg). This data has been previously reported [14] where the antioxidant capacity of isorhamnetin glycosides was found to be 3.2-6.1 times the activity of the isorhamnetin aglycone. The ORAC antioxidant method is an indicator of the peroxyl radical scavenging capacity of antioxidants and measures their hydrogen atom donating ability (HAT-based method).

In the TEAC antioxidant method, isorhamnetin glycosides IG1, IG2 and IG4 all showed high antioxidant capacity values (123-140 $\mu \mathrm{mol}$ Trolox eq./mg). Meanwhile, indicaxanthin, betanin, ascorbic acid and piscidic acid all showed the lowest antioxidant capacity (6-9 $\mu$ mol Trolox eq./mg). The TEAC antioxidant method measures the ability of antioxidants to scavenge the stable radical cation ABTS ${ }^{+}$ by single electron transfer (SET-based method).

\section{Putative Antioxidant Mechanisms of Betalains in the LOX-FL Assay}

Soybean lipoxygenase (LOX)-1 assays are based on the aerobic and anaerobic reaction of the enzyme in the presence of linoleic acid. A key role in LOX-1 catalysis is played by non-heme iron atom, cycling from the oxidized form (III) to the reduced one (II). When the main aerobic cycle consumes oxygen in the reaction mixture, the anaerobic cycle starts and different physiological reactive species, including the peroxyl (LOO•), alkoxyl (LO•), hydroxyl $(\bullet \mathrm{OH})$ and alkylic (L•) radicals as well as the singlet oxygen $\left({ }^{1} \mathrm{O}_{2}\right)$ are generated. These oxidant species induce the bleaching of fluorescein. Interestingly, the soybean LOX-1-catalysed FL-bleaching may be delayed, inhibited or even prevented by antioxidants acting according to different mechanisms, such as the capacity to scavenge one or more free radical species, as well as other antioxidant mechanisms involving chelating or reducing activities of iron ion essential for LOX-1 catalysis, singlet oxygen quenching, hydroperoxide decomposition, and direct inhibition of pro-oxidative LOX-1 apo-enzyme.

Betanin and betanidin (betacyanins) inhibit the primary aerobic reaction of LOX-1 isoenzyme at very low concentrations, with $\mathrm{IC}_{50}$ values of 0.25 and $0.5 \mu \mathrm{M}$, respectively [7]. This suggests that the strong inhibition observed by betalains in the LOX-FL assay was achieved through reduction of iron to the ferrous inactive form and/or by interacting with the enzyme peroxyl radical complex. This data agrees with our 
results reported in Fig. 2A in which a marked increase of lag phase of the LOX-FL reaction due to betanin is shown. A similar mechanism could be supposed for indicaxanthin (betaxanthin) due to its similar chemical structure which has also shown ferric reducing antioxidant power [22]. In the light of their putative reducing power, betanin and indicaxanthin could inhibit LOX-1 also at level of the anaerobic cycle, where a key role of non-heme iron atom in LOXcatalysis is also played.

Moreover, efficient antioxidant action against LOO', LO, ${ }^{1} \mathrm{O}_{2}, \cdot \mathrm{OH}$ and the ability to prevent lipid peroxidation when incorporated into liposomal bilayers submitted to AAPH, have been also reported for betanin [7-9] and indicaxanthin [10]. This data agrees with the findings in Fig. 2A-B where, in the presence of betanin and indicaxanthin, a significant reduction in the rate of fluorescein bleaching can be observed. Interestingly, the presence of glycosylation and further acylation in the chemical structures of betalains have been reported to reduce the radical scavenging activity mainly of betacyanins (such as betanin) [23]. This evidence is further supported by our results by the LOX-FL method (Table 1), which highlight a higher antioxidant capacity for indicaxanthin than for betanin.

\section{Comparison of the Antioxidant Capacity in Extracts by LOX-FL, ORAC and TEAC Methods}

The antioxidant capacity of peel and pulp extracts from $O$. ficus-indica and O. stricta var. Dillenii fruits were evaluated by the LOX-FL spectrophotometric protocol and compared to the widely used ORAC and TEAC assays (Fig. 3; Supplementary Table S4). TEAC and ORAC antioxidant assays mainly assess the reducing power capacity and scavenging activity against peroxyl radicals, respectively; whereas the LOX-FL method simultaneously detects scavenging capacity against different physiological radicals as well as other antioxidant functions, thus providing a more comprehensive result on antioxidant capacity $[18,23]$.

The LOX-FL, ORAC and TEAC antioxidant assays provided different results for aqueous extracts of Opuntia spp. fruits in the light of their different mechanisms of detection. Interestingly, all three assays agreed in highlighting a significant higher antioxidant efficacy for pulp extracts from O. stricta var. Dillenii fruit tissues. However, tendencies in peels were significantly different in the LOX-FL assay compared to ORAC and TEAC antioxidant methods. The antioxidant capacity in prickly pear peels by ORAC and TEAC was highest in the fruits with the highest phenolic content (Colorada and Blanca $O$. ficus-indica fruits), particularly isorhamnetin glycosides. In the LOX-FL method of peels, $O$. stricta var. Dillenii had the highest antioxidant capacity due to its high betanin content. Meanwhile, $O$. ficus-indica varieties had higher antioxidant capacity measured by TEAC
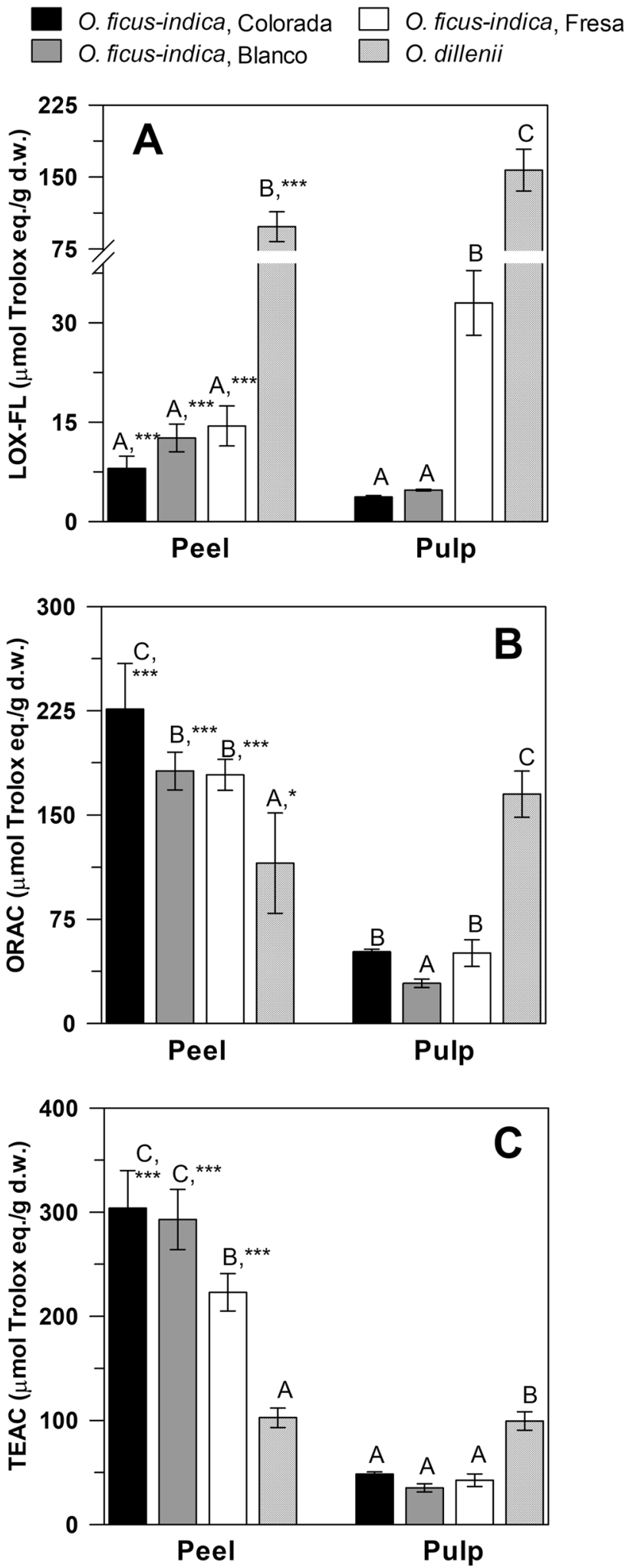

Fig. 3 Antioxidant capacity of Opuntia spp. fruit extracts by (A) LOX-FL, (B) ORAC and (C) TEAC methods. Letters indicate statistically significant differences $(p<0.01)$ between genotypes. $*$, *** indicate the probability level at $P \leq 0.05$ and $P \leq 0.001$, respectively, relative to the comparison between peel and pulp values. Raw data may be consulted in Supplementary Table S4 
and ORAC antioxidant methods. It should be emphasized that the $O$. ficus-indica fruit varieties generally showed a higher antioxidant performance in peels than pulps (with the only exception of Fresa measured by LOX-FL). While in $O$. dilleni, the antioxidant effectiveness of the peel was lower (measured by LOX-FL and ORAC), or at most similar, (measured by TEAC) than its pulp.

\section{Correlation Analysis Between Antioxidant Capacity and Antioxidant Content in Extracts}

Pearson's correlation between the antioxidant capacity of all Opuntia spp. peel and pulp tissues by LOX-FL, ORAC and TEAC assays and their bioactive content (individual betalains, phenolic compounds and ascorbic acid) are shown in Supplementary Table S5.

The antioxidant capacity of Opuntia spp. extracts determined by the LOX-FL method had strong correlation with betanin $(\mathrm{r}=0.90)$, isobetanin $(\mathrm{r}=0.93)$, and total betalain $(\mathrm{r}=0.89)$ content at $p \leq 0.01$. However, no significant correlation was found between the LOX-FL method and indicaxanthin content, possibly due to the scarce indicaxanthin-rich O. ficus-indica varieties included in the study (only Colorada). We suggest further studies comparing the antioxidant capacity of several indicaxanthin-rich plant foods by the LOX-FL reaction.

Both ORAC and TEAC assays correlated $(p \leq 0.01)$ with phenolic compounds, namely, piscidic acid, IG1, IG2 and IG4. ORAC and TEAC methods can detect hydrogen atom transfer and single electron transfer, respectively, which are well-established antioxidant mechanisms for phenolic compounds. However, ORAC and TEAC antioxidant methods did not correlate with betalains in Opuntia spp. fruits, despite their abundance. In the literature, there are inconsistent results regarding the correlation of these assays with betalain content.

None of the antioxidant assays used in this study correlated with ascorbic acid content. Nevertheless, in the light of its high reducing and radical scavenging capacities, ascorbic acid could contribute to prickly pears' antioxidant capacity by protecting other antioxidant constituents from oxidative damage.

As for comparison among the different antioxidant capacity assays, a positive correlation $(0.895, p \leq 0.01)$ was found between TEAC and ORAC results, whereas the LOX-FL method appeared unrelated to ORAC and TEAC.

\section{Conclusions}

The correlation between antioxidant activity by the LOX-FL assay and betalain content suggest the effectivity of this in vitro method to reflect the in vivo antioxidant capacity of betalains. This is attributable to the capability of these compounds to affect the aerobic and anaerobic reactions involved in the LOX-FL assay: i) inhibition of LOX-1 through conversion to its ferrous inactive form and interaction with the enzyme-peroxyl radical-complex, and ii) scavenging of peroxyl, alkoxyl, hydroxyl radicals. We expect this method could be used in the future to analyze the antioxidant activity of betalain-rich foods because of its biological relevance.

Supplementary Information The online version contains supplementary material available at https://doi.org/10.1007/s11130-021-00914-7.

Acknowledgements We thank the funding from Spanish Ministry of Science and Innovation (Spain), projects INIA RTA201500044-C02-02 and PID2020-118300RB-C21. We thank Gloria Lobo (ICIA) for the recollection and provision of prickly pear fruits (RTA2015-00044-C02-01). Andrea Gómez-Maqueo thanks CONACyT (Mexico) for her doctoral scholarship 692751.

Funding Open Access funding provided thanks to the CRUE-CSIC agreement with Springer Nature.

Data Availability All data generated or analyzed during this study are included in this published article and its supplementary information files.

\section{Declarations}

Conflicts of Interest The authors declare that there are no conflicts of interest.

Open Access This article is licensed under a Creative Commons Attribution 4.0 International License, which permits use, sharing, adaptation, distribution and reproduction in any medium or format, as long as you give appropriate credit to the original author(s) and the source, provide a link to the Creative Commons licence, and indicate if changes were made. The images or other third party material in this article are included in the article's Creative Commons licence, unless indicated otherwise in a credit line to the material. If material is not included in the article's Creative Commons licence and your intended use is not permitted by statutory regulation or exceeds the permitted use, you will need to obtain permission directly from the copyright holder. To view a copy of this licence, visit http://creativecommons.org/licenses/by/4.0/.

\section{References}

1. Azeredo HM (2009) Betalains: properties, sources, applications, and stability-a review. Int J Food Sci 44(12):2365-2376

2. Angulo-Bejarano PI, Gómez-García MDR, Valverde ME, ParedesLópez O (2019) Nopal (Opuntia spp.) and its effects on metabolic syndrome: new insights for the use of a millenary plant. Curr Pharm Des 25(32):3457-3477

3. Gómez-Maqueo A, Antunes-Ricardo M, Welti-Chanes J, Cano MP (2020) Digestive stability and bioaccessibility of antioxidants in prickly pear fruits from the Canary Islands: healthy foods and ingredients. Antioxidants 9(2):164

4. Gómez-Maqueo A, García-Cayuela T, Fernández-López R, WeltiChanes J, Cano MP (2019) Inhibitory potential of prickly pears and their isolated bioactives against digestive enzymes linked 
to type 2 diabetes and inflammatory response. J Sci Food Agric 99(14):6380-6391

5. Tran TMT, Thanh BN, Moussa-Ayoub TE, Rohn S, Jerz G (2019) Profiling of polar metabolites in fruits of Opuntia stricta var. dillenii by ion-pair high-performance countercurrent chromatography and off-line electrospray mass-spectrometry injection. J Chromatogr A 1601:274-287

6. Betancourt C, Cejudo-Bastante MJ, Heredia FJ, Hurtado N (2017) Pigment composition and antioxidant capacity of betacyanins and betaxanthins fractions of Opuntia dillenii (Ker Gawl) Haw cactus fruit. Food Res Int 101:173-179

7. Kanner J, Harel S, Granit R (2001) Betalains a new class of dietary cationized antioxidants. J Agric Food Chem 49(11):5178-5185

8. Wendel M, Nizinski S, Gierszewski M, Prukala D, Sikorski M, Starzak K, Wybraniec S, Burdzinski G (2016) Chemical quenching of singlet oxygen by betanin. Photoch Photobio Sci 15(7):872-878

9. Esatbeyoglu T, Wagner AE, Motafakkerazad R, Nakajima Y, Matsugo S, Rimbach G (2014) Free radical scavenging and antioxidant activity of betanin: electron spin resonance spectroscopy studies and studies in cultured cells. Food Chem Toxicol 73:119-126

10. Tesoriere L, Allegra M, Butera D, Gentile C, Livrea MA (2007) Kinetics of the lipoperoxyl radical-scavenging activity of indicaxanthin in solution and unilamellar liposomes. Free Radic Res 41(2):226-233

11. Allegra M, Furtmüller PG, Jantschko W, Zederbauer M, Tesoriere L, Livrea MA, Obinger C (2005) Mechanism of interaction of betanin and indicaxanthin with human myeloperoxidase and hypochlorous acid. Biochem Bioph Res Co 332(3):837-844

12. Hadipour E, Fereidoni M, Tayarani-Najaran Z (2020) Betanin attenuates oxidative stress induced by 6-OHDA in PC12 cells via SAPK/JNK and PI3 K pathways. Neurochem Res 45(2):395-403

13. Martinez RM, Hohmann MS, Longhi-Balbinot DT, Zarpelon AC, Baracat MM, Georgetti SR, Vicentini FTMC, Sassonia RC, Verri WA, Casagrande R (2020) Analgesic activity and mechanism of action of a Beta vulgaris dye enriched in betalains in inflammatory models in mice. Inflammopharmacology 28(6):1663-1675

14. Pastore D, Laus MN, Tozzi D, Fogliano V, Soccio M, Flagella Z (2009) New tool to evaluate a comprehensive antioxidant activity in food extracts: bleaching of 4-nitroso-N, N-dimethylaniline catalyzed by soybean lipoxygenase-1. J Agric Food Chem 57(20):9682-9692
15. Gandía-Herrero F, García-Carmona F, Escribano J (2016) Development of a protocol for the semi-synthesis and purification of betaxanthins. Phytochem Anal 17(4):262-269

16. García-Cayuela T, Gómez-Maqueo A, Guajardo-Flores D, WeltiChanes J, Cano MP (2019) Characterization and quantification of individual betalain and phenolic compounds in Mexican and Spanish prickly pear (Opuntia ficus-indica L. Mill) tissues: a comparative study. J Food Compost Anal 76:1-13

17. Santos-Zea L, Gutiérrez-Uribe JA, Serna-Saldivar SO (2011) Comparative analyses of total phenols, antioxidant activity, and flavonol glycoside profile of cladode flours from different varieties of Opuntia spp. J Agric Food Chem 59(13):7054-7061

18. Soccio M, Laus MN, Alfarano M, Pastore D (2016) The soybean lipoxygenase-fluorescein reaction may be used to assess antioxidant capacity of phytochemicals and serum. Anal Methods 8(22):4354-4362

19. Laus MN, Gagliardi A, Soccio M, Flagella Z, Pastore D (2012) Antioxidant activity of free and bound compounds in quinoa (Chenopodium quinoa Willd.) seeds in comparison with durum wheat and emmer. J Food Sci 77(11):C1150-C1155

20. Ou B, Hampsch-Woodill M, Prior RL (2001) Development and validation of an improved oxygen radical absorbance capacity assay using fluorescein as the fluorescent probe. J Agric Food Chem 49(10):4619-4626

21. Gonçalves LC, Lopes NB, Augusto FA, Pioli RM, Machado CO, Freitas-Dörr BC, Suffredini HB, Bastos EL (2020) Phenolic betalain as antioxidants: meta means more. Pure Appl Chem 92(2):243-253

22. Taira J, Tsuchida E, Katoh MC, Uehara M, Ogi T (2015) Antioxidant capacity of betacyanins as radical scavengers for peroxyl radical and nitric oxide. Food Chem 166:531-536

23. Soccio M, Laus MN, Flagella Z, Pastore D (2018) Assessment of antioxidant capacity and putative healthy effects of natural plant products using soybean lipoxygenase-based methods: an overview. Molecules 23(12):3244

Publisher's Note Springer Nature remains neutral with regard to jurisdictional claims in published maps and institutional affiliations. 